\title{
Facile, Economic, and Size-Tunable Synthesis of Metal Arsenide Nanocrystals
}

\author{
Vishwas Srivastava, ${ }^{\dagger}$ Eric M. Janke, ${ }^{\dagger}$ Benjamin T. Diroll, ${ }^{\ddagger}$ Richard D. Schaller, ${ }^{\ddagger}, \S$ and \\ Dmitri V. Talapin $*,+, \ddagger$
}

$\dagger$ Department of Chemistry and James Frank Institute, University of Chicago, Illinois 60637, United States

$\$$ Center for Nanoscale Materials, Argonne National Lab, Argonne, Illinois 60439, United States

$\S_{\text {Department of Chemistry, Northwestern University, Evanston, Illinois 60208, United States }}$

E-mail: dvtalapin@uchicago.edu 
Supporting Tables and Figures:

Table S1. Synthetic parameters used to produce InAs NCs. (red color indicates different reaction conditions, s.f. $=$ size fractioned).

\begin{tabular}{|c|c|c|c|c|c|}
\hline $\begin{array}{l}\mathrm{InCl}_{3} \\
\text { (mmol) }\end{array}$ & $\begin{array}{l}\mathrm{As}\left(\mathrm{NMe}_{2}\right)_{3} \\
\text { (mmol) }\end{array}$ & $\begin{array}{l}\text { DIBAL-H } \\
(\mathbf{1 M}) \\
(\mathbf{m L})\end{array}$ & $\begin{array}{l}\text { Temp } \\
\left({ }^{\circ} \mathrm{C}\right)\end{array}$ & $\begin{array}{l}\text { Time } \\
\text { (mins) }\end{array}$ & $\begin{array}{l}\lambda_{\text {abs }} \\
(\mathbf{n m})\end{array}$ \\
\hline 0.2 & 0.2 & 1.2 & 240 & 3 & 760 \\
\hline 0.2 & 0.2 & 1.2 & 240 & 5 & 800 \\
\hline 0.2 & 0.2 & 1.2 & 240 & 50 & 860 \\
\hline 0.2 & 0.2 & 1.2 & 280 & 15 & 960 \\
\hline 0.2 & 0.2 & $1.2,0.6$ & 280,240 & 60,10 & 1050 \\
\hline 0.2 & 0.2 & $0.6,1.2$ & 260,240 & 15,100 & 1100 \\
\hline 0.4 & 0.4 & 1 & 280 & 10 & 1165 (s.f.) \\
\hline 0.2 & 0.2 & 0.2 & 240 & 5 & 1250 \\
\hline 0.4 & 0.4 & 0.4 & 280 & 5 & 1330 \\
\hline 0.4 & 0.4 & 0.4 & 280 & 15 & 1400(s.f.) \\
\hline 0.4 & 0.4 & 0.4 & 280 & 15 & 1450 (s.f.) \\
\hline
\end{tabular}

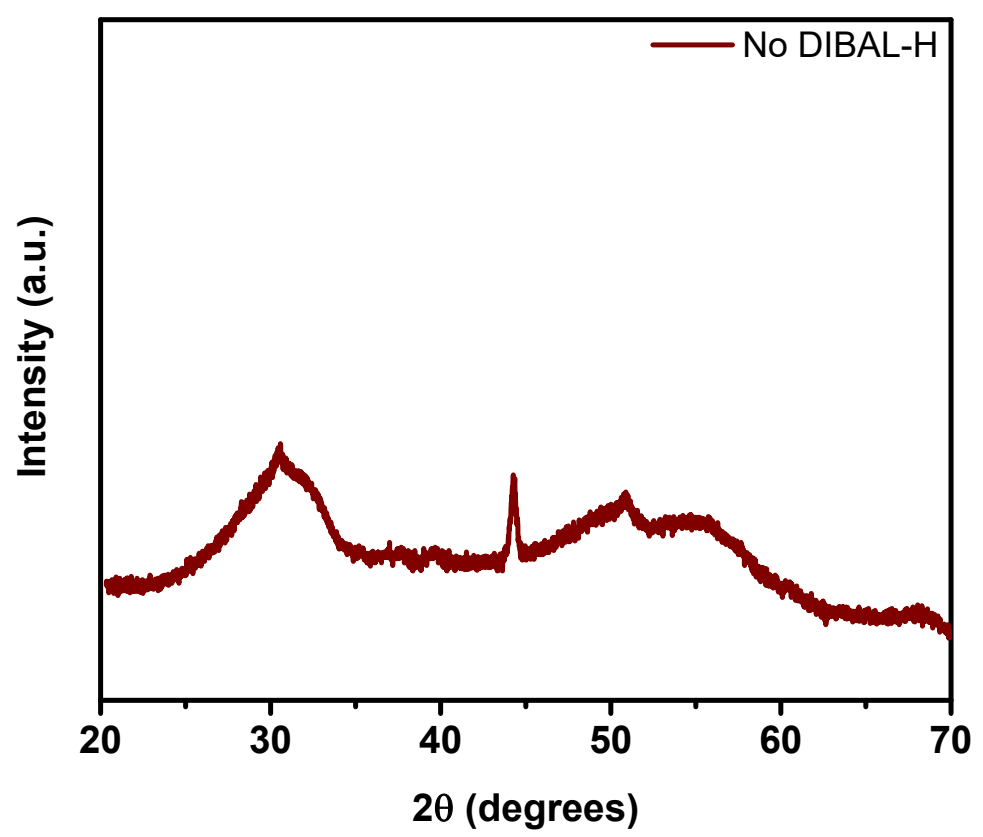

Figure S1. Powder XRD pattern of a reaction between $\mathrm{InCl}_{3}$ and $\mathrm{As}\left(\mathrm{NMe}_{2}\right)_{3}$ in oleylamine in the absence of DIBAL-H. 

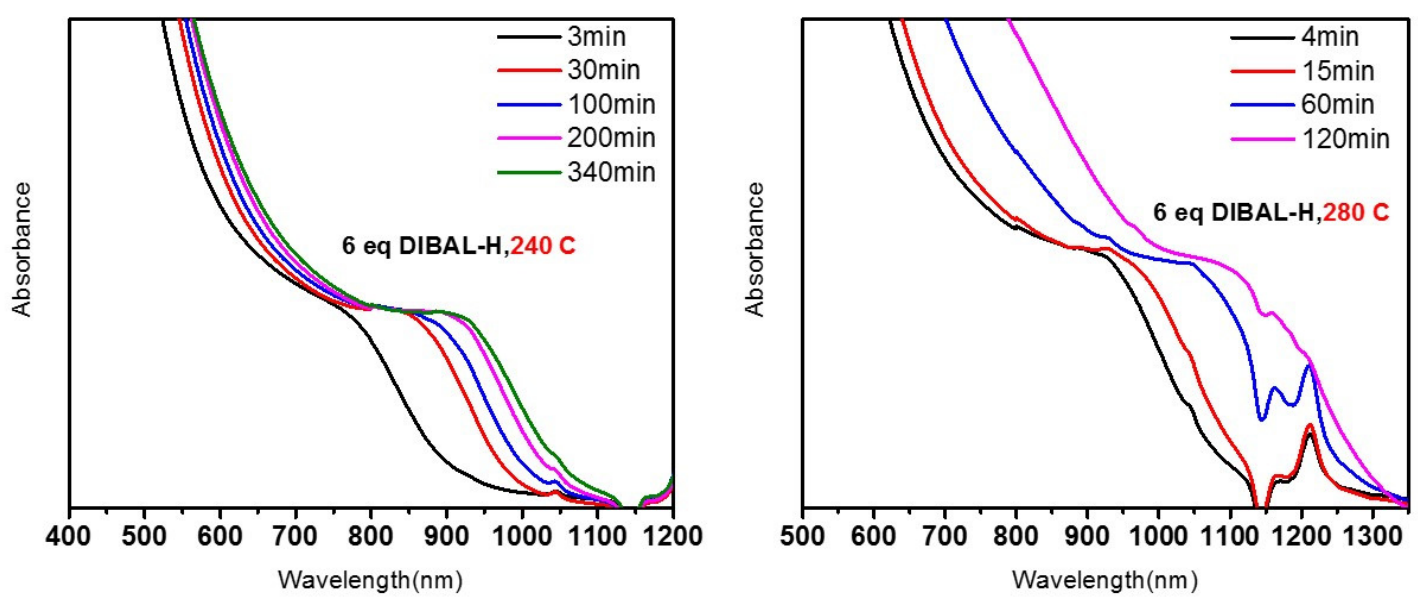

Figure S2. Effect of Temperature on the growth of InAs NCs at two different reaction temperatures. Absorption spectra of aliquots taken at regular time intervals.
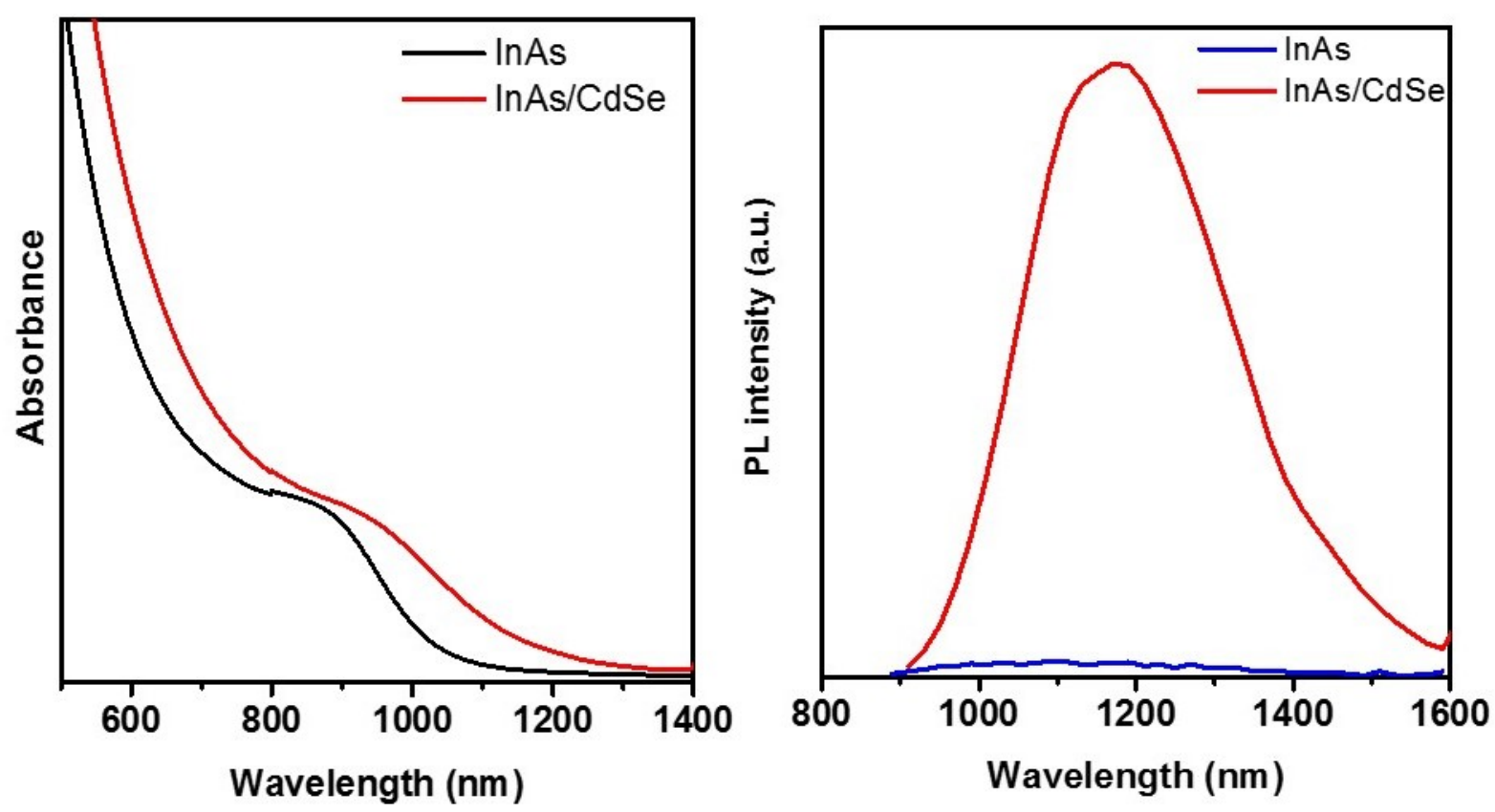

Figure S3. Characterization of InAs/CdSe core-shell NCs. (Left) Absorption spectra of $\sim 3 \mathrm{~nm}$ InAs cores and InAs/CdSe core shells. (Right) PL spectra of InAs cores and InAs/CdSe Core shell NCs showing $\sim 100$ fold increase in PL efficiency upon shell growth. 

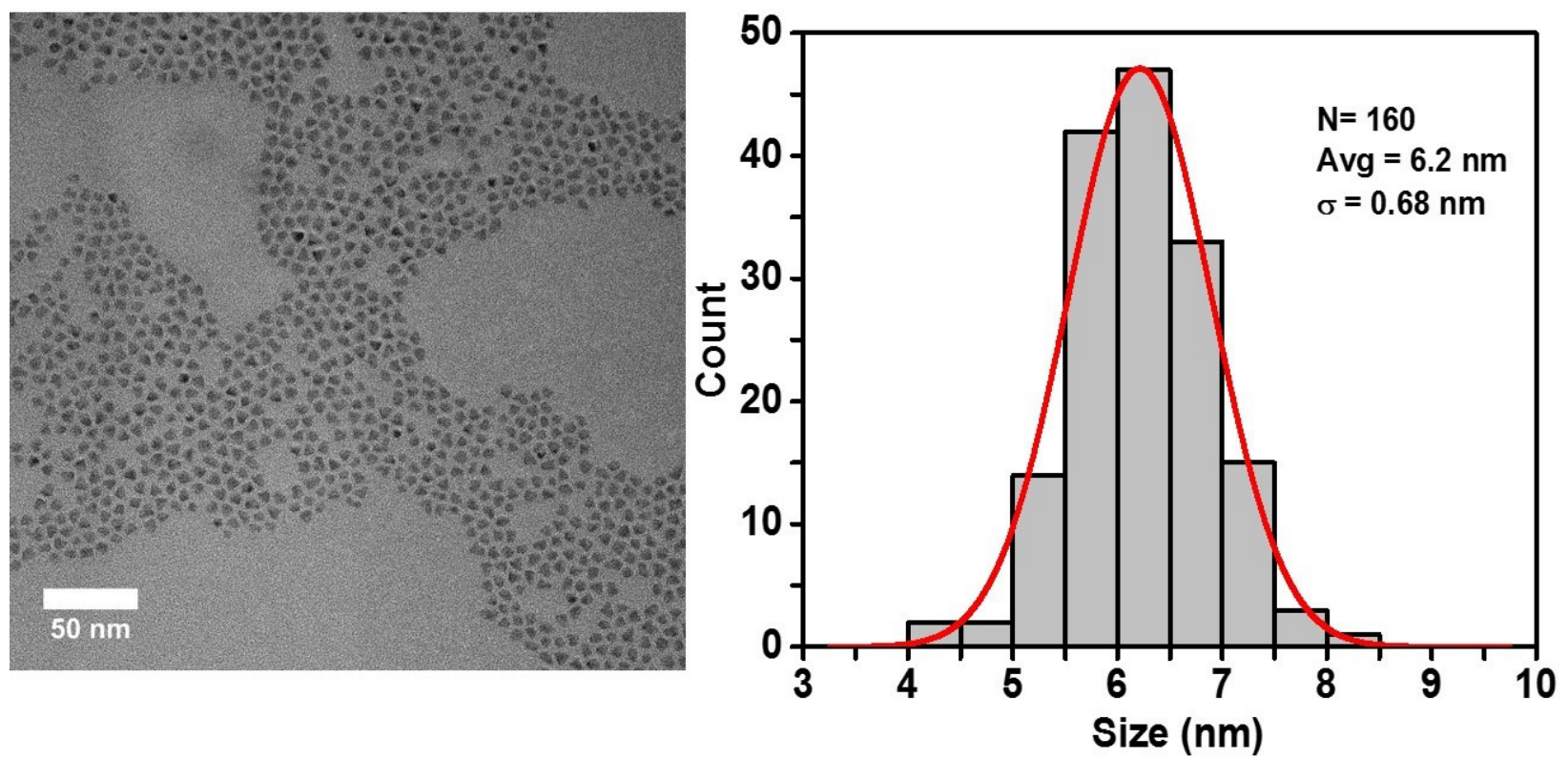

Figure S4. A representative TEM image of InAs NCs and the size-distribution histogram
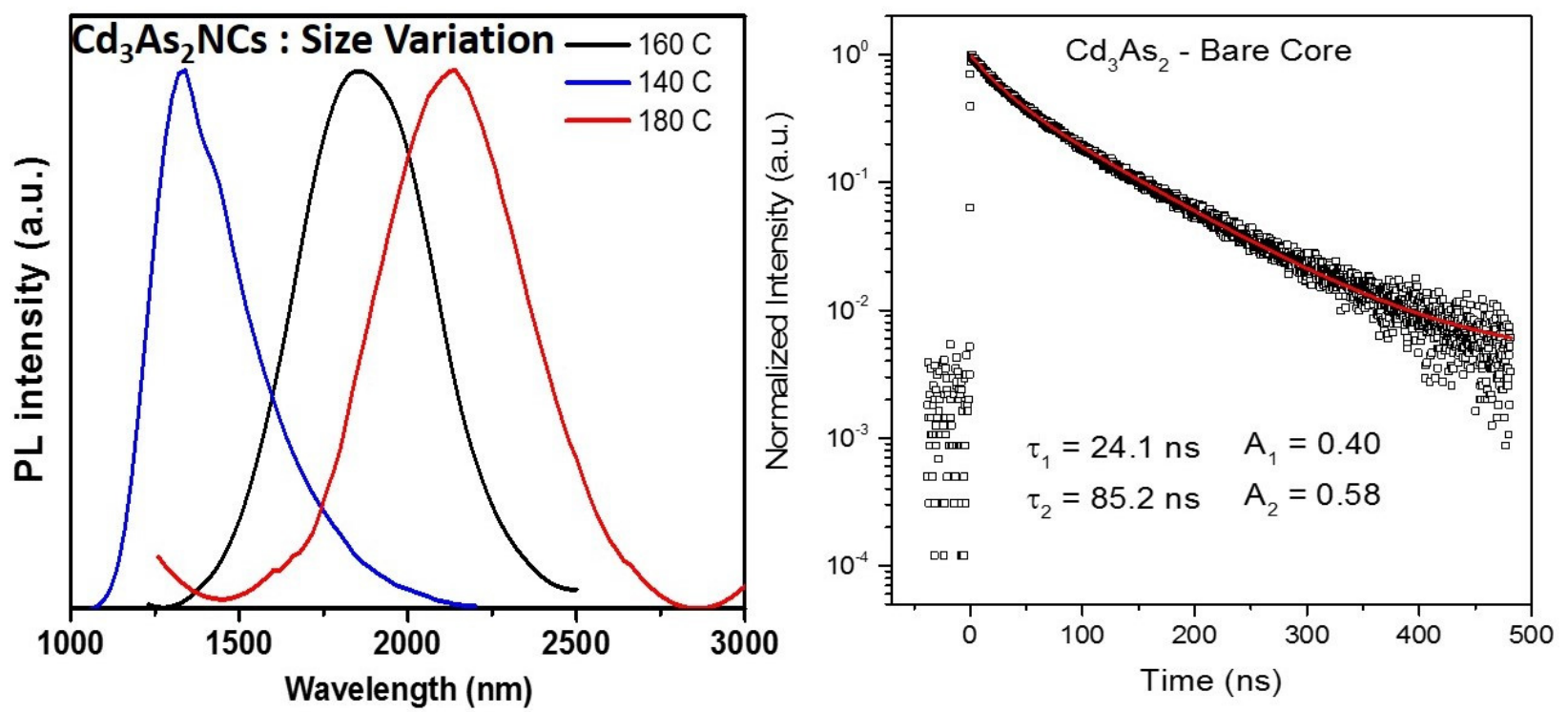

Figure S5. PL spectra of $\mathrm{Cd}_{3} \mathrm{As}_{2} \mathrm{NCs}$ grown at different temperature and time-resolved PL decay of small $\mathrm{Cd}_{3} \mathrm{As}_{2} \mathrm{NCs}$ (corresponding to blue curve in the left panel) 

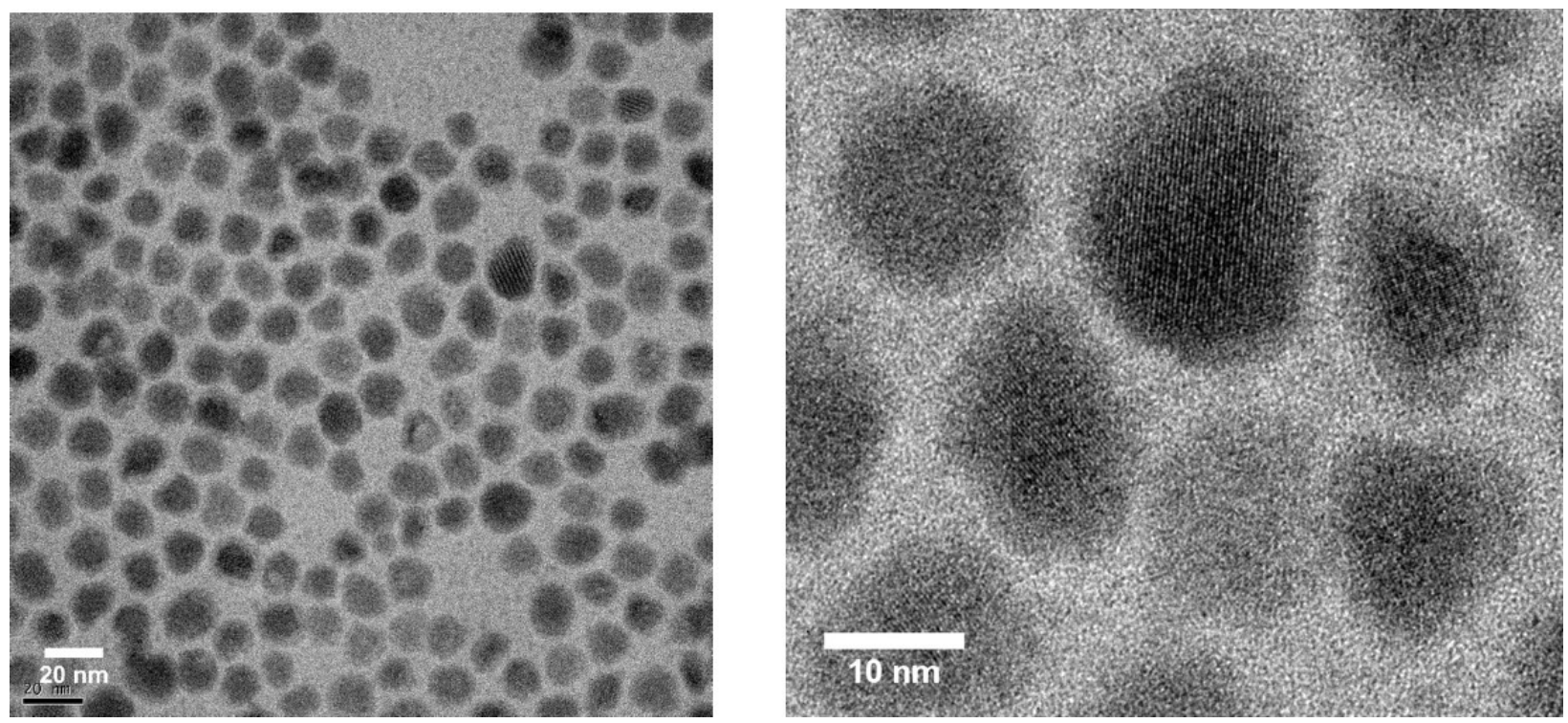

Figure S6. TEM image showing large $\mathrm{Cd}_{3} \mathrm{As}_{2} \mathrm{NCs}(>10 \mathrm{~nm})$ and an HRTEM image of $\mathrm{Cd}_{3} \mathrm{As}_{2}$ NCs. These NCs were grown at a temperature of $220 \mathrm{C}$ for 2 mins with 3 equivalents of DIBAL$\mathrm{H}$ with respect to $\mathrm{As}\left(\mathrm{NMe}_{2}\right)_{3}$. 